\title{
Impacto na qualidade de vida de famílias com crianças e adolescentes com transtornos mentais
}

\section{Impact on the quality of life of family with children and adolescents with mental disorders}

\author{
Francisco de Brito Melo Júnior ${ }^{1}$. Verônica Maria Gomes de Carvalho². Eugênio de Moura Campos ${ }^{1,2}$. \\ 1 Universidade Federal do Ceará (UFC), Fortaleza, Ceará, Brasil. 2 Hospital Universitário Walter Cantídio (HUWC/UFC/ \\ EBSERH), Fortaleza, Ceará, Brasil.
}

\section{RESUMO}

Objetivo: Avaliar o impacto na qualidade de vida ligada à saúde em pais de crianças e adolescentes portadores de transtornos mentais em um ambulatório de psiquiatria de um hospital pediátrico e relacionar com os dados sociodemográficos. Método: Trata-se de um estudo transversal, quantitativo e descritivo realizado na cidade de Fortaleza-CE, no período de setembro e outubro de 2018. A população do estudo foi composta pelos pais dos pacientes que responderam o questionário sociodemográfico e o inventário PedsQL - Modulo de Impacto na Família, Versão2.0. Resultados: Os dados dos 57 participantes foram avaliados e se obteve média de 67,8 com desvio padrão de 29,8 na pontuação total. Houve interações significativas entre o PedsQL e dados sobre os pais: o estado civil e renda familiar; em relação ao paciente: idade, religião, fazer uso de medicação, estar em acompanhamento psicoterápico, idade do início do transtorno mental, idade em que iniciou tratamento medicamentoso e a concordância entre o diagnóstico declarado pelos pais e o registrado no prontuário. Conclusão: $O$ estudo revelou dados que podem ser úteis para o planejamento de ações e cuidados dos pacientes e familiares e na prática clínica do SUS.

Palavras-chave: Saúde mental. Qualidade de vida. Psiquiatria infantil. Psiquiatria do adolescente.

\section{ABSTRACT}

Objective: To evaluate the impact on health-related quality of life in parents of children and adolescents with mental disorders in a psychiatric outpatient clinic of a pediatric hospital and to relate with their sociodemographic data. Method: This is a crosssectional, quantitative and descriptive study carried out in the city of Fortaleza, CE, in the period of September and October of 2018. The study population was composed by the parents of the patients who answered the sociodemographic questionnaire and the PedsQL inventory - Family Impact Module, Version 2.0. Results: The data of the 57 participants were evaluated and a mean of 67.8 with a standard deviation of 29.8 was obtained in the total score. There were significant interactions between the PedsQL and data on the parents: marital status and family income; in relation to the patient: age, religion, use of medication, psychotherapeutic follow-up, age of onset of the mental disorder, age at which drug treatment began, and agreement between the diagnosis declared by the parents and the registered in the medical record. Conclusion: The study revealed data that may be useful for planning actions and care of patients and families, and in the clinical practice of SUS.

Keywords: Mental health. Quality of life. Child psychiatry. Adolescent psychiatry.

Autor correspondente: Francisco de Brito Melo Júnior, Rua Mato Grosso, 720, Shopping Rio Poty, Porenquanto, Teresina, Piaui. CEP: $64000-710$. Telefone: +55 86 99821-2420. E-mail: psiquiatria@outlook.com

Conflito de interesses: Não há qualquer conflito de interesses por parte de qualquer um dos autores.

Recebido em: 27 Mar 2020; Revisado em: 16 Out 2020; Aceito em: 04 Mai 2021. 


\section{INTRODUÇÃO}

A Organização das Nações Unidas (ONU) há algum tempo ressalta a importância dos transtornos mentais na infância e adolescência. ${ }^{1}$ Segundo o Instituto Brasileiro de Geografia e Estatística (IBGE), em 2018, a população brasileira de pessoas de 0-19 anos de idade era de $29,21 \%$ do total. ${ }^{2}$ Dentro dessa faixa etária estima-se que pelo menos 10 a $20 \%$ sofram de transtornos mentais e que desses pelo menos $3 \%$ a $4 \%$ precisem de tratamento relativo a saúde mental. ${ }^{3}$ Os transtornos emocionais e comportamentais já são considerados as doenças crônicas mais comuns enfrentadas pelos clínicos gerais em ambientes médicos gerais para crianças e adolescentes nos países desenvolvidos e entre os mais comuns nos países em desenvolvimento. ${ }^{4}$

Em condições crônicas, os tratamentos frequentes, sintomas e possíveis problemas na autoimagem são desencadeadores de diversos sentimentos como medo, vergonha, tristeza e dúvidas. ${ }^{5}$ Além disso, essas condições geram um impacto individual por afetar as atividades diárias e a longo prazo prejudicam o crescimento e desenvolvimento do paciente. ${ }^{6}$

Há também um impacto na família pois o cuidador passa a lidar diariamente com comportamentos imprevisíveis da criança, gastos financeiros consideráveis, frustração de expectativas sociais e sofrem com o estigma social relacionado ao adoecimento psíquico. ${ }^{7}$

A concepção ampliada criada pela Organização Mundial da Saúde (OMS) de que "Saúde é o estado do mais completo bem-estar físico, mental e social e não apenas a ausência de enfermidade" superou o que antes era medido apenas pela mortalidade. Dessa forma, novas medidas foram desenvolvidas e aplicadas para aferir o grau em que determinadas doenças comprometem a vida dos indivíduos. ${ }^{8}$

A Qualidade de Vida Ligada a Saúde (QVLS) é um termo que engloba um conceito mais amplo de saúde. Considera que quase todos os aspectos da vida podem se relacionar a saúde, como por exemplo a renda, a liberdade e a qualidade do ambiente em que se vive. A QVLS é, dessa forma, importante para medir o impacto da doença crônica, pois pacientes em condições clínicas semelhantes podem ter um bem-estar emocional diferente. ${ }^{9}$

O Módulo de Impacto na Família (MIF) do Inventário de Qualidade de Vida Pediátrica (PedsQL 4.0) é uma das poucas medidas de avaliação do impacto das condições de saúde crônicas pediátricas na qualidade de vida ligada a saúde dos pais e no funcionamento familiar disponíveis. O que o torna diferente das outras ferramentas é a sua estrutura multidirecional pois avalia 8 áreas específicas, além de apresentar uma escala de Funcionamento em Família, que avalia o grau em que a doença crônica afeta o sistema familiar em termos de atividades de vida diária e conflitos ou tensão entre os membros da família. A outra vantagem é o fato de ser de fácil aplicação. ${ }^{10}$

A literatura, durante a realização do estudo, era abundante em estudos utilizando o MIF do PedsQL 4.0 para avaliar familiares de pacientes portadores de doenças crônicas orgânicas como esclerose múltipla, hérnia diafragmática congênita, asma, dermatomiosite, entre outras. ${ }^{9-21}$ No entanto, eram poucos os artigos que tinham utilizado o MIF em familiares de pacientes portadores de transtornos mentais. Em uma busca na literatura foram encontrados apenas três artigos. ${ }^{22-24}$ Além disso, nenhum desses três trabalhos abordou pacientes de um ambulatório de saúde mental de forma geral, eles se limitam a estudar familiares de pacientes portadores de transtornos específicos.

O estudo buscou avaliar o impacto na qualidade de vida de pais de crianças e adolescentes portadores de transtornos mentais, descrever o perfil sociodemográfico da população do estudo e identificar a presença de interações estatísticas entre as dimensões da qualidade de vida dos pais com os dados sociodemográficos dos pais e dos pacientes e com dados clínicos dos pacientes.

\section{MATERIAL E MÉTODOS}

Trata-se de um estudo do tipo transversal, quantitativo e descritivo. Os participantes foram pais e mães que estavam acompanhando seus filhos em consulta médico psiquiátrica no ambulatório de psiquiatria do Hospital Infantil Albert Sabin (HIAS), integrante da rede de assistência da secretaria de saúde do estado do Ceará, localizado na cidade de Fortaleza. A amostra foi realizada por conveniência e incluiu pais que aceitaram participar de forma voluntária e assinaram termo de consentimento livre e esclarecido.

Como critérios de inclusão para composição da amostra foram considerados pais e mães legalmente constituídos de pacientes que compareceram à consulta no ambulatório de psiquiatria do HIAS acompanhando o paciente no período do estudo, que já tinham passado por pelo menos uma consulta psiquiátrica anterior, que tinham diagnóstico em prontuário e que estavam em tratamento para o transtorno.

Foram excluídos pais de pacientes ainda em processo de investigação do diagnóstico e pais com algum tipo de déficit cognitivo (constatado ou suspeito) que impossibilitava o entendimento das perguntas dos questionários.

Os dados foram coletados nos meses de setembro e outubro de 2018 no horário de funcionamento do ambulatório de psiquiatria do HIAS. Os questionários utilizados foram lidos aos participantes em virtude do baixo grau de instrução da maioria. A participação ocorreu mediante a assinatura do Termo de Consentimento Livre e Esclarecido, formalizando a concordância da participação do integrante.

Perguntas referentes a um questionário sociodemográfico e dados clínicos e um instrumento de avaliação da qualidade de vida foram aplicados aos participantes. Os pais responderam primeiro o questionário sociodemográfico. Este foi composto por dados relativos a: idade, sexo, escolaridade, estado civil, renda familiar e religião dos pais; dados sociodemográficos do paciente: idade, sexo e religião; dados clínicos do paciente: diagnóstico do transtorno psiquiátrico, diagnóstico de outra 
doença crônica, uso atual de medicação, qual medicação fazia uso, idade do diagnóstico psiquiátrico, idade do início do tratamento do transtorno psiquiátrico e participação atual de acompanhamento psicoterápico. O diagnóstico e as medicações em uso declarados pelos pais posteriormente foram comparados com os presentes no prontuário da última consulta médica.

$\mathrm{O}$ instrumento de qualidade de vida utilizado foi o MIF do PedsQL 4.0 que é destinado a avaliar o impacto das condições crônicas de saúde dos filhos na QVLS dos pais e no Funcionamento em Família (FF). O MIF consiste em um total de 36 itens. O instrumento avalia, em cada item, a frequência ( 0 = "nunca"; 1 = "quase nunca"; 2 = "algumas vezes"; 3 = "frequentemente"; 4 = "quase sempre") das situações ocorridas para o pai/mãe durante o mês passado. O MIF estima a QVLS referida pelos pais e o impacto familiar em 8 áreas: funcionamento físico (6 itens), funcionamento emocional (5 itens), funcionamento social (4 itens), funcionamento cognitivo (5 itens), comunicação (3 itens), preocupação (5 itens), atividades diárias (3 itens), e relacionamentos familiares (5 itens). Uma pontuação geral do impacto total é calculada pela média de todos os 36 itens. Uma pontuação parcial da QVLS é calculada pela média dos 20 itens que compões as escalas de funcionamento físico, emocional, social e cognitivo. A pontuação parcial do FF é calculada pela média dos 8 itens que compõem as áreas de atividades diárias e relações familiares. Os itens da MIF são pontuados de maneira reversa e transformados linearmente em uma escala de 0 a 100 , de modo que os escores mais altos indicam melhor QVLS ou menor impacto familiar negativo. ${ }^{23}$ O MIF foi validado para o Português do Brasil por Scarpelli ${ }^{11}$ e explorado por pesquisadores de todo o mundo em diversas doenças crônicas.
O projeto foi aprovado pelo comitê de ética do Hospital Albert Sabin, CAAE: 96904818.8 .0000 .5042 e número do parecer: 2.951.643.

Para a correção dos instrumentos citados, foram utilizados os crivos de correção do próprio instrumento. Os dados obtidos através do inventário foram analisados e categorizados através do software de processamento de dados "Statistical Package for Social Sciences" (SPSS) for Windows, versão 18.0, seguido de formatação, armazenamento de informações e tratamento estatístico.

\section{RESULTADOS}

A população do estudo foi composta por 57 pessoas. $\mathrm{Na}$ Tabela 1 foram descritos os dados da distribuição sociodemogáfica em número absoluto e em razão de porcentagem. Os resultados mostraram predominância do sexo feminino $(n=52)$ entre os acompanhantes. A renda das famílias era majoritariamente igual a um salário mínimo $(63,2 \%)$. A maior parte $(52,6 \%)$ era constituída por pessoas entre as quais o maior nível de escolaridade era o ensino fundamental. Observou-se que a maioria (76,8\%) deles declarou diagnóstico do filho não concordante com o registrado no prontuário.

A Tabela 2 discrimina as interações significativas encontradas após análise estatística dos dados sociodemogáficos e clínicos relacionadas a pontuações parciais e total do MIF. Para a análise da pontuação total foram utilizados 56 questionários respondidos, visto que em um deles faltaram ser preenchidos alguns dados. Obteve-se média de 67,8 e mediana de 63,5 nos escores do MIF. O desvio padrão dos escores do MIF foi de 29,8 .

Tabela 1. Distribuição sociodemogáfica da população do estudo.

\begin{tabular}{|c|c|c|c|}
\hline Variável & & $\mathrm{n}$ & $\%$ \\
\hline \multirow[t]{2}{*}{ Sexo do pai/mãe } & Feminino & 52 & 91,2 \\
\hline & Masculino & 5 & 8,8 \\
\hline \multirow[t]{2}{*}{ Estado Civil do pai/mãe } & Com companheiro(a) & 40 & 70,2 \\
\hline & Sem companheiro(a) & 17 & 30,8 \\
\hline \multirow[t]{5}{*}{ Escolaridade do pai/mãe } & Analfabeto & 5 & 8,8 \\
\hline & Ensino Fundamental & 30 & 52,6 \\
\hline & Ensino Médio & 20 & 35,1 \\
\hline & Ensino Superior & 2 & 3,5 \\
\hline & Pós-graduação & 0 & 0 \\
\hline \multirow[t]{3}{*}{ Renda aproximada da família em salários mínimos } & $<1$ & 11 & 19,3 \\
\hline & 1 & 36 & 63,2 \\
\hline & 2 ou mais & 10 & 17,5 \\
\hline \multirow[t]{2}{*}{ Pai/mãe é praticante de alguma religião } & Não & 11 & 19,3 \\
\hline & Sim & 46 & 80,7 \\
\hline \multirow[t]{2}{*}{ Sexo do paciente } & Feminino & 12 & 21,1 \\
\hline & Masculino & 45 & 78,9 \\
\hline
\end{tabular}

Continua 
Conclusão.

Tabela 1. Distribuição sociodemogáfica da população do estudo.

\begin{tabular}{|c|c|c|c|}
\hline Variável & & $\mathrm{n}$ & $\%$ \\
\hline \multirow[t]{2}{*}{ O paciente é praticante de alguma religião } & Não & 14 & 24,6 \\
\hline & Sim & 43 & 75,4 \\
\hline \multirow[t]{2}{*}{ O paciente é portador de alguma doença crônica não psiquiátrica } & Não & 37 & 64,9 \\
\hline & $\operatorname{Sim}$ & 20 & 35,1 \\
\hline \multirow[t]{2}{*}{ O paciente faz uso de algum medicamento de forma contínua } & Não & 2 & 3,5 \\
\hline & Sim & 55 & 96,5 \\
\hline \multirow[t]{2}{*}{ O paciente faz acompanhamento psicoterápico } & Não & 35 & 61,4 \\
\hline & $\operatorname{Sim}$ & 21 & 36,8 \\
\hline \multirow[t]{2}{*}{ Diagnóstico declarado concordante com o prontuário } & Não & 43 & 76,8 \\
\hline & $\operatorname{Sim}$ & 13 & 23,2 \\
\hline \multirow[t]{2}{*}{ Tratamento medicamentoso declarado concordante com o prontuário } & Não & 26 & 46,4 \\
\hline & Sim & 30 & 53,6 \\
\hline
\end{tabular}

Tabela 2. Interações significativas entre o PedsQL e os dados sociodemográficos e clínicos.

\begin{tabular}{|c|c|c|c|c|c|c|}
\hline Dados & PedsQL & Variáveis & $\mathrm{p}$ & Mediana/CC & P25 & P75 \\
\hline \multirow[t]{6}{*}{ Estado civil $^{1}$} & \multirow[t]{2}{*}{ Capacidade física } & Com & 0,005 & $\mathrm{M}=8,00$ & 5,00 & 12,00 \\
\hline & & Sem & & $M=15,00$ & 10,00 & 18,00 \\
\hline & \multirow[t]{2}{*}{ QVLS } & Com & 0,013 & $\mathrm{M}=1,50$ & 0,95 & 1,95 \\
\hline & & Sem & & $\mathrm{M}=2,30$ & 1,65 & 2,85 \\
\hline & \multirow[t]{2}{*}{ Total } & Com & 0,044 & $M=60,00$ & 41,00 & 81,00 \\
\hline & & Sem & & $\mathrm{M}=86,00$ & 57,00 & 100,00 \\
\hline \multirow{4}{*}{$\begin{array}{l}\text { Renda da família em } \\
\text { salários mínimos }{ }^{1}\end{array}$} & \multirow{4}{*}{$\begin{array}{l}\text { Aspecto social } \\
\mathrm{p}=0,009\end{array}$} & $>=2$ & 0,013 & $\mathrm{M}=0$ & 0 & 3,00 \\
\hline & & 1 & & $\mathrm{M}=6,00$ & 2,00 & 11,50 \\
\hline & & $>=2$ & 0,013 & $\mathrm{M}=0$ & 0 & 3,00 \\
\hline & & $<1$ & & $\mathrm{M}=8,00$ & 3,00 & 12,00 \\
\hline Idade do paciente ${ }^{2}$ & Preocupação & & 0,032 & $\mathrm{CC}=0,284$ & & \\
\hline \multirow{4}{*}{$\begin{array}{l}\text { Paciente é praticante } \\
\text { de religião }{ }^{1}\end{array}$} & \multirow[t]{2}{*}{ Aspecto social } & Não & 0,015 & $\mathrm{M}=8,50$ & 6,00 & 12,00 \\
\hline & & Sim & & $\mathrm{M}=4,00$ & 0 & 10,00 \\
\hline & \multirow[t]{2}{*}{ QVLS } & Não & 0,045 & $\mathrm{M}=2,08$ & 1,25 & 2,85 \\
\hline & & Sim & & $\mathrm{M}=1,60$ & 0,95 & 2,05 \\
\hline \multirow{10}{*}{$\begin{array}{l}\text { Paciente faz uso de } \\
\text { medicação de forma } \\
\text { contínua? }^{3}\end{array}$} & \multirow[t]{2}{*}{ Capacidade física } & Não & 0,026 & $\mathrm{M}=1,00$ & 0 & 2,00 \\
\hline & & Sim & & $\mathrm{M}=9.50$ & 6,00 & 15,00 \\
\hline & \multirow[t]{2}{*}{ Aspecto emocional } & Não & 0,003 & $\mathrm{M}=0$ & 0 & 0 \\
\hline & & Sim & & $M=9,00$ & 4,00 & 14,00 \\
\hline & \multirow[t]{2}{*}{ Comunicação } & Não & 0,038 & $\mathrm{M}=1,00$ & 0 & 2,00 \\
\hline & & Sim & & $\mathrm{M}=5,00$ & 3,00 & 6,00 \\
\hline & \multirow[t]{2}{*}{ QVLS } & Não & 0,003 & $\mathrm{M}=0,23$ & 0,20 & 0,25 \\
\hline & & $\operatorname{Sim}$ & & $\mathrm{M}=1,67$ & 1,00 & 2,45 \\
\hline & \multirow[t]{2}{*}{ Total } & Não & 0,008 & $M=22,50$ & 20,00 & 25,00 \\
\hline & & Sim & & $M=65,50$ & 48,00 & 91,00 \\
\hline
\end{tabular}

Legenda: CC: coeficiente de contingência; M: mediana; QVLS: qualidade de vida ligada a saúde; FF: funcionamento e família; P25: percentil 25; P75: percentil 75; 'Teste de Kruskal-Wallis de amostras independentes; ${ }^{2}$ Correlação de Spearman; ${ }^{3}$ Teste U de Mann-Whitney.

Continua. 
Conclusão.

Tabela 2. Interações significativas entre o PedsQL e os dados sociodemográficos e clínicos.

\begin{tabular}{|c|c|c|c|c|c|c|}
\hline Dados & PedsQL & Variáveis & $\mathrm{p}$ & Mediana/CC & $\mathrm{P} 25$ & P75 \\
\hline \multirow{10}{*}{$\begin{array}{l}\text { Paciente está fazendo } \\
\text { acompanhamento } \\
\text { psicoterápico? }{ }^{3}\end{array}$} & \multirow[t]{2}{*}{ Capacidade física } & Não & \multirow[t]{2}{*}{0,045} & $\mathrm{M}=8,00$ & 4,00 & 13,00 \\
\hline & & Sim & & $\mathrm{M}=14,00$ & 8,00 & 10,00 \\
\hline & \multirow[t]{2}{*}{ Capacidade mental } & Não & \multirow[t]{2}{*}{0,034} & $\mathrm{M}=8,00$ & 2,00 & 14,00 \\
\hline & & Sim & & $M=10,00$ & 6,00 & 17,00 \\
\hline & \multirow[t]{2}{*}{ Comunicação } & Não & \multirow[t]{2}{*}{0,040} & $\mathrm{M}=4,00$ & 2,00 & 6,00 \\
\hline & & Sim & & $\mathrm{M}=6,00$ & 3,00 & 8,00 \\
\hline & \multirow[t]{2}{*}{ QVLS } & Não & \multirow[t]{2}{*}{0,040} & $\mathrm{M}=1,55$ & 0,75 & 2,15 \\
\hline & & Sim & & $\mathrm{M}=1,95$ & 1,35 & 2,65 \\
\hline & \multirow[t]{2}{*}{ Total } & Não & \multirow[t]{2}{*}{0,043} & $\mathrm{M}=58,50$ & 39,00 & 81,00 \\
\hline & & Sim & & $\mathrm{M}=78,00$ & 56,00 & 100,00 \\
\hline \multirow{5}{*}{$\begin{array}{l}\text { Idade em que o } \\
\text { paciente recebeu } \\
\text { diagnóstico do } \\
\text { transtorno mental? }{ }^{2}\end{array}$} & \multicolumn{2}{|l|}{ Aspecto emocional } & 0,001 & $\mathrm{CC}=-0,427$ & & \\
\hline & \multicolumn{2}{|l|}{ Aspecto social } & 0,003 & $\mathrm{CC}=-0,288$ & & \\
\hline & \multicolumn{2}{|l|}{ QVLS } & 0,015 & $\mathrm{CC}=-0,323$ & & \\
\hline & \multicolumn{2}{|l|}{ FF } & 0,039 & $C C=-0,274$ & & \\
\hline & \multicolumn{2}{|l|}{ Total } & 0,017 & $\mathrm{CC}=-0,318$ & & \\
\hline $\begin{array}{l}\text { Idade em que o paciente } \\
\text { iniciou tratamento do } \\
\text { transtorno mental? }{ }^{2}\end{array}$ & \multicolumn{2}{|l|}{ Aspecto emocional } & 0,014 & \multicolumn{2}{|l|}{$\mathrm{CC}=-0,324$} & \\
\hline \multirow{2}{*}{$\begin{array}{l}\text { Diagnóstico } \\
\text { declarado } \\
\text { concordante com o } \\
\text { prontuário? }\end{array}$} & \multirow[t]{2}{*}{ Preocupações } & Não & \multirow[t]{2}{*}{0,007} & $\mathrm{M}=15,00$ & 12,50 & 16,50 \\
\hline & & Sim & & $\mathrm{M}=12,00$ & 10,00 & 13,00 \\
\hline
\end{tabular}

Legenda: CC: coeficiente de contingência; M: mediana; QVLS: qualidade de vida ligada a saúde; FF: funcionamento e família; P25: percentil 25; P75: percentil 75; ${ }^{1}$ Teste de Kruskal-Wallis de amostras independentes; ${ }^{2}$ Correlação de Spearman; ${ }^{3}$ Teste U de Mann-Whitney.

O estado civil dos pais apresentou interação significativa com a pontuação total do $\operatorname{MIF}(p=0,044)$. Nesse caso a mediana da pontuação total foi melhor para os pais e mães sem companheiro $(\mathrm{m}=86,00)$ do que com companheiro $(\mathrm{m}=$ $60,00)$. Também foram observadas diferenças significativas semelhantes na pontuação QVLS (com companheiro $\mathrm{m}=1,50$; sem companheiro $m=2,30 ; p=0,013)$ e na capacidade física (com companheiro $\mathrm{m}=8,00$; sem companheiro $\mathrm{m}=15,00 ; \mathrm{p}=$ $0,005)$ (Tabela 2).

A renda da família mostrou associação estatisticamente significante $(p=0,009)$ com a área aspecto social do MIF. Houve melhor pontuação nas famílias que recebiam um salário mínimo ou menos de um salário mínimo comparando com quem recebia dois ou mais salários mínimos. Foi identificada correlação significativa positiva entre a idade do paciente e o item específico - preocupação - do inventário $(\mathrm{CC}=0,284$, $\mathrm{p}=$ 0,032).

Foi identificada interação significativa entre melhor pontuação da QVLS $(p=0,045)$ e do aspecto social $(p=0,015)$ dos pais de crianças e adolescentes que não eram religiosos praticantes (Tabela 2).
Houve significância estatística também na interação encontrada entre o paciente fazer uso de medicação de forma contínua e os itens capacidade física $(\mathrm{p}=0,026)$, aspecto emocional $(p=0,003)$, comunicação $(p=0,038)$, QVLS $(p=$ $0,003)$ e a pontuação total $(p=0,008)$ (Tabela 2). Já entre o fato do paciente fazer acompanhamento psicoterápico, a associação de significância se deu com capacidade física $(p=0,014)$, capacidade mental $(p=0,034)$, comunicação $(p=0,040)$, QVLS $(p=0,040)$ e a pontuação total $(p=0,043)$ (Tabela 2).

Correlações significativas também estavam presentes entre a idade em que o paciente recebeu o diagnóstico do transtorno mental e aspecto emocional $(p=0,001)$, aspecto social $(p=$ $0,003)$, QVLS $(p=0,015), F F(p=0,039)$ e a pontuação total do MIF $(p=0,017)$ (Tabela 2). Essas correlações foram negativas, de modo que, quanto menor a idade em que foi feito o diagnóstico, maior a pontuação.

Em temos do início precoce do tratamento, este também se associou à melhor pontuação no inventário. No entanto, a correlação significativa aconteceu apenas no aspecto social $(\mathrm{p}=0,014)$ do MIF (Tabela 2). 
Além disso houve interação significativa entre o diagnóstico declarado não concordante com o prontuário e a área do MIF denominada preocupação $(p=0,007)$ (Tabela 2).

\section{DISCUSSÃO}

A amostra foi composta por pais e mães que acompanhavam seus filhos no ambulatório de psiquiatria de um hospital geral. Verificou-se a predominância de mulheres entre esses acompanhantes, concordante com o estudo de Muylaert, Delfini e Reis que mostrou que dentre os acompanhantes de um ambulatório de psiquiatria, a mulher era a principal cuidadora, mesmo nos casos em que ela era a responsável pela renda. $^{25}$

A renda das famílias avaliadas no ambulatório era majoritariamente igual a um salário mínimo. Apesar de não ter sido calculado o número de pessoas por domicílio e assim ser possível obter o valor da renda per capita, estima-se que esse valor esteja abaixo do que era encontrado como renda familiar per capita média no Ceará em 2017, aproximadamente 824 reais. ${ }^{26}$

A busca na literatura com a finalidade de identificar trabalhos semelhantes a este resultou apenas em três estudos que utilizaram o MIF do PedsQL em familiares de pacientes portadores de transtornos mentais: Limbers et al., ${ }^{23}$ Hsieh, Hsieh e Lee ${ }^{24}$ e Hsieh et al. ${ }^{22}$

Limbers et al. utilizaram o MIF, mas avaliaram somente familiares de crianças portadoras do transtorno do déficit de atenção e hiperatividade (TDAH). Eles buscaram comparar a pontuação total do MIF em uma clínica pediátrica geral com a de uma clínica infantil psiquiátrica. Na clínica psiquiátrica foi obtida a média de 53,96 com o desvio padrão (DP) de 19,20 e na clínica geral média de 69,56 ( $\mathrm{DP}=19,86)$. Dessa forma, demostraram que os familiares dos pacientes que eram atendidos no ambulatório geral tinham em média menor impacto na qualidade de vida do que os atendidos no ambulatório psiquiátrico. Este achado provavelmente deve-se ao fato de que pacientes atendidos em ambulatório não especializado tendem a ser menos graves. ${ }^{23}$ Os dados do presente trabalho, realizado em um ambulatório de psiquiatria em um hospital pediátrico geral, resultaram em uma média de 67,80 , número mais próximo ao encontrado por eles no ambulatório geral, mas com um desvio padrão maior (DP = 29,80).

Hsieh, Hsieh e Lee em 2016, delineou o trabalho buscando comparar os scores do MIF de pais de crianças com atraso no desenvolvimento antes e depois de algumas sessões de terapia em grupo. O valor obtido da média do score total do MIF antes das terapias foi de $62,4(\mathrm{DP}=11,8)$ e após média de 79,0 (DP $=10,3) \cdot{ }^{24}$ Os dados do trabalho aqui detalhados foram maiores do que os aferidos antes e menores do que os aferidos depois pelos referidos autores, mas com maior desvio padrão.

A pontuação total do MIF foi maior para os pais e mães sem companheiro do que com companheiro. O mesmo foi observado nos escores da QVLS e da capacidade física.
Pode-se supor que na casa de genitores com companheiro(a) havia uma ou mais variáveis não exploradas no estudo, que tenham influenciado o resultado, como por exemplo conflitos conjugais e violência doméstica.

Houve melhor pontuação do aspecto social nas famílias que recebiam um salário mínimo ou menos de um salário mínimo comparando com quem recebia dois ou mais salários mínimos. A melhor condição financeira não se associou a melhora na qualidade de vida, contudo, novamente há a possibilidade de ter ocorrido, concomitantemente com a renda, um fator não avaliado neste estudo que tenha influenciado os dados, como os benefícios sociais que algumas das famílias recebiam em virtude da condição de saúde dos seus filhos.

Não foi observada interação significativa entre a idade do paciente e a pontuação total do MIF. Porém houve interação significativa positiva na parte específica do inventário preocupação, isto é, quanto maior a idade, maior a pontuação no item, o que representa, de acordo com o inventário, menos preocupação dos pais. Já o estudo de Hsieh et al. em 2009, mostrou correlação significativa negativa entre a idade do paciente e o impacto na qualidade de vida dos pais. ${ }^{22}$ Cabe ressaltar que o estudo de Hsieh et. al utilizou o teste de Pearson para a comparação dos questionários e este trabalho utilizou o teste de Rô de Spearman.

No campo dos transtornos mentais, já foi demonstrado que religião/espiritualidade $(\mathrm{R} / \mathrm{E})$ tem implicações na prevalência, diagnóstico, tratamento, desfecho e prevenção, bem como na qualidade de vida. Embora haja evidências mostrando que $\mathrm{R} / \mathrm{E}$ está geralmente associada a melhores resultados na saúde, também pode trazer prejuizos tais como recusa de tratamento, intolerância, coping religioso negativo. ${ }^{27}$ Aqui foi identificada melhor pontuação da QVLS e do aspecto social nos pais das crianças e adolescentes que não eram religiosos praticantes.

No quesito tratamento, os pais cujos filhos faziam uso contínuo de medicação obtiveram melhor pontuação em capacidade física, aspecto emocional, comunicação, QVLS e na pontuação total do MIF. Não foram encontrados na literatura, estudos sobre avalição da qualidade de vida de familiares de pacientes com transtornos mentais relacionados ao uso de medicação. Há um estudo de Varni et al., que estudaram o efeito do aripiprazol no tratamento da irritabilidade em crianças com transtorno autista, utilizando o inventário PedsQL “Generic Core”, versão diferente do PedsQL, que é aplicada diretamente ao paciente. Os achados sugeriram que o uso desta medicação está associado com melhora da QVLS. ${ }^{28}$ O presente estudo mostrou que as famílias cujos pacientes faziam uso de medicação tinham uma melhor pontuação para a qualidade de vida. Porém, apesar de significativa a associação, os dados apresentam um viés, visto que 96,5\% da amostra fazia uso de medicação. Em relação ao fato do paciente estar em acompanhamento psicoterápico, os escores melhores se deram em capacidade física, capacidade mental, comunicação, QVLS e na pontuação total do MIF.

Quanto à idade em que o paciente recebeu o diagnóstico do transtorno mental, correlações negativas estavam presentes em 
aspecto emocional, aspecto social, QVLS, FF e a pontuação total do MIF, de modo que, quanto menor a idade em que foi feito o diagnóstico, melhor a pontuação. $\mathrm{O}$ início precoce do tratamento também se associou a melhor pontuação total do MIF, no entanto a associação significativa aconteceu apenas no aspecto social. Isso mostra a necessidade de se fazer o diagnóstico precoce dos transtornos psiquiátricos e assim poder iniciar o tratamento o mais cedo possível.

Ademais, buscou-se verificar se havia um correto entendimento do transtorno dos filhos por parte dos pais. A maioria declarou diagnóstico não concordante com o registrado no prontuário e apresentou um escore mais elevado no item preocupação do inventário, o que corresponde a menos preocupação. Tendo em conta a baixa escolaridade da amostra, é factível levantar a hipótese de que este fator pudesse comprometer uma satisfatória compreensão de prejuízos que os pacientes poderiam vir a ter no futuro, não implicando em maiores preocupações.

Sumarizando os achados, o estudo evidenciou que a pontuação total do MIF, a QVLS e o item capacidade física foram maiores para os pais e mães sem companheiro(a); houve melhor pontuação do aspecto social nas famílias que recebiam um salário mínimo ou menos; mostraram menos preocupação os pais de pacientes com maior idade e aqueles que declararam

\section{REFERÊNCIAS}

1.World Health Organization. The World Health Report 2001, mental health: new understanding, new Hope [Internet]. Geneva: World Health Organization; 2001 [acesso em: 29 abril 2019]. Disponível em: https://www.who.int/whr/2001/en/whr01_en.pdf?ua=1

2.Brasil. Instituto Brasileiro de Geografia e Estatística. Projeção da população do Brasil e das Unidades da Federação [Internet]. [place unknown]: IBGE; [acesso em: 29 abril 2019]. Disponível em: https:// www.ibge.gov.br/apps/populacao/projecao/

3.Flaviana OP, Cerqueira MB. Saúde mental infanto-juvenil: usuários e suas trajetórias de acesso aos serviços de saúde mental. Revista Espaço para a Saúde. 2008;10(1): 34-46.

4.Kelleher K. Prevention and intervention in primary care. In: Remschmidt H, Belfer ML, Goodyer I, eds. Facilitating pathways: care, treatment and prevention in child and adolescent mental health. Berlin: Springer; 2004. p. 313-25.

5.Nobrega RD, Collet N, Gomes IP, Holanda ER, Araújo YB. Criança de idade escolar hospitalizada: significado da condição crônica. Texto contexto - enfermagem. 2010;19(3):425-33.

6.Silva MD, Collete N, Silva KD, Moura FM. Cotidiano da família no enfrentamento da condição crônica na infância. Acta Paulista de Enfermagem. 2010;23(3):359-65.

7.Monteiro AR, Teixeira LA, Silva RS, Rabelo KP, Tavares SF, Távora RC. Sofrimento psíquico em crianças e adolescentes - a busca pelo tratamento. Esc Anna Nery. 2012;16(3):523-9.

8.Alvarenga TM, Caldeira AP. Qualidade de vida em pacientes diagnóstico diferente do registrado no prontuário; os pais das crianças e adolescentes que não eram religiosos praticantes tiveram melhor pontuação na QVLS e no aspecto social; tratamento medicamentoso continuado e acompanhamento psicoterápico dos pacientes implicaram na melhora total do MIF e de diversos aspectos específicos da qualidade de vida; quanto menor a idade em que o diagnóstico foi estabelecido, melhor a pontuação total do MIF e, tanto mais precoce o início do tratamento, melhor o item aspecto social.

Em consonância com os princípios do Sistema Único de Saúde (SUS), o planejamento das ações para promoção, proteção e recuperação da saúde deve partir das necessidades de saúde da população. A compreensão das realidades locais abrange o levantamento de um amplo conjunto de indicadores sociodemográficos, epidemiológicos, sanitários, de educação, culturais, ocupacionais, entre outros, os quais devem embasar a tomada de decisões pelos gestores. ${ }^{29}$ Sendo assim, espera-se que os dados obtidos por meio deste trabalho possam ser úteis para o planejamento de ações e cuidados dos pacientes e familiares e na prática clínica do SUS e que também ensejem a realização de novos estudos a fim de explorar hipóteses, esclarecer dados emergentes e avançar na compreensão dos fenômenos envolvidos na determinação da qualidade de vida relacionada à saúde de familiares e pacientes.

pediátricos com dermatite atópica. J Pediatr (Rio J). 2009;85(5):41520 .

9.Guyatt GH, Feeny DH, Patrick DL. Measuring health-related quality of life. Ann Intern Med. 1993;118:622-9.

10.Mano KE, Khan KA, Ladwig RJ, Weisman SJ. The impact of pediatric chronic pain on parents' health-related quality of life and family functioning: reliability and validity of the PedsQL 4.0 Family Impact Module. Journal of Pediatric Psychology. 2011;36:517-27.

11.Scarpelli AC, Paiva SM, Pordeus IA, Varni JW, Viegas CM, Allison PJ. The pediatric quality of life inventoryTM (PedsQLTM) family impact module: reliability and validity of the Brazilian version. Health Qual Life Outcomes. 2008;6:35.

12.Guimaraes CA, Enumo SR. Impacto familiar nas diferentes fases da leucemia infantil. Psicologia Teoria e Prática. 2015;17(3):66-78.

13.Fritz KA, Khmour AY, Kitzerow K, Sato TT, Basir M. Healthrelated quality of life, educational and family outcomes in survivors of congenital diaphragmatic hernia. Pediatr Surg Int. 2019;35(3):315-20.

14.Taminskiene V, Alasevicius T, Valiulis A, Vaitkaitiene E, Stukas R, Hadjipanayis A, et al. Quality of life of the family of children with asthma is not related to asthma severity. Eur J Pediatr. 2019;178(3):369-76.

15.Psihogios AM, Daniel LC, Tarazi R, Smith-Whitley K, Patterson CA, Barakat LP. Family functioning, medical self-management, and health outcomes among school-aged children with sickle cell 
disease: a mediation model. Journal of Pediatric Psychology. 2018;43:423-33.

16. Johaningsmeir SA, Colby H, Krauthoefer M, Simpson P, Conceição SC, Gordon JB. Impact of caring for children with medical complexity and high resource use on family quality of life. J Pediatr Rehabil Med. 2015;8:75-82.

17. Mishra K, Ramachandran S, Firdaus S, Rath B. The impact of pediatric nephrotic syndrome on parents' health-related quality of life and family functioning: an assessment made by the PedsQL 4.0 Family Impact Module. Saudi J Kidney Dis Transpl. 2015;26:285-92.

18. McGowan JE, Alderdice FA, Boylan J, Holmes VA, Jenkins J, Craig S, et al. Neonatal intensive care and late preterm infants: health and family functioning at three years. Early Hum Dev. 2014;90(4):201-5.

19.Mazaheri MM, Rae-Seebach RD, Preston HE, Schmidt M, Kountz-Edwards S, Field N, et al. The impact of Prader-Willi syndrome on the family's quality of life and caregiving, and the unaffected siblings' psychosocial adjustment. Journal of Intellectual Disability Research. 2013;57:861-73.

20.Huang IC, Anderson M, Gandhi P, Tuli S, Krull K, Lai JS, et al. The relationships between fatigue, quality of life, and family impact among children with special health care needs. Journal of Pediatric Psychology. 2013;38(7):722-31.

21.Isa SN, Aziz AA, Rahman AA, Ibrahim MI, Ibrahim WP, Mohamad N, et al. The impact of disabled children on parent healthrelated quality of life and family functioning in Kelantan and its associated factors. J Dev Behav Pediatr. 2013;34(4):262-8.

22.Hsieh RL, Huang HY, Lin MI, Wu CW, Lee W. Quality of life, health satisfaction and family impact on caregivers of children with developmental delays. Child Care Health Dev. 2009;35:243-9.

23.Limbers CA, Ripperger-Suhler J, Boutton K, Ransom D, Varni JW. A comparative analysis of health-related quality of life and family impact between children with ADHD treated in a general pediatric clinic and a psychiatric Clinic Utilizing the PedsQL. J Atten Disord. 2010;15(5):392-402.

24. Hsieh RL, Hsieh WH, Lee WC. Short-term family-centered workshop for children with developmental delays enhances family functioning and satisfaction: a prospective clinical trial. Medicine. 2016;95(31):e4200.

25. Muylaert CJ, Delfini PS, Reis AO. Relações de gênero entre familiares cuidadores de crianças e adolescentes de serviços de saúde mental. Physis. 2015;25(1)41-58.

26.Brasil. Lei ${ }^{\circ}$. 8.742, de 7 de dezembro de 1993. Dispõe sobre a organização da Assistência Social e dá outras providências. Brasília: Diário Oficial da União; 1993 [acesso em: 29 abril 2019]. Disponível em: http://www.planalto.gov.br/ccivil_03/leis/18742.htm

27.Moreira-Almeida A, Sharma A, Janse van Rensburg B, Verhagen PJ, Cook CC. WPA position statement on spirituality and religion in psychiatry. World Psychiatry. 2016;15(1):87-8.

28. Varni JW, Handen BL, Corey-Lisle PK, Guo Z, Manos G, Ammerman DK, et al. Effect of aripiprazole 2 to $15 \mathrm{mg} / \mathrm{d}$ on healthrelated quality of life in the treatment of irritability associated with autistic disorder in children: a post hoc analysis of two controlled trials. Clin Ther. 2012;34(4):980-92.

29. Brasil. Ministério da Saúde. Manual de planejamento no SUS. Brasília: Ministério da Saúde; 2016.

\section{Como citar:}

Melo FB Júnior, Carvalho VM, Campos EM. Impacto na qualidade de vida de famílias com crianças e adolescentes com transtornos mentais. Rev Med UFC. 2021;61(1):1-8. 